\title{
Anti-windup controller design for singularly perturbed systems subject to actuator saturation
}

ISSN 1751-8644

Received on 3rd March 2015

Revised on 13th August 2015 Accepted on 1st November 2015 doi: 10.1049/iet-cta.2015.0189 www.ietdl.org

\author{
Chunyu Yang ${ }^{1, 凶, ~ L i n g l i ~ Z h a n g ~}{ }^{1}$, Jing Sun ${ }^{2}$ \\ ${ }^{1}$ School of Information and Electrical Engineering, China University of Mining and Technology, Xuzhou 221116, People's Republic of China \\ ${ }^{2}$ Department of Naval Architecture \& Marine Engineering, University of Michigan Ann Arbor, MI 48109, USA \\ $\bowtie$ E-mail: chunyuyang@cumt.edu.cn
}

\begin{abstract}
This study considers the problem of anti-windup (AW) controller design for singularly perturbed systems with actuator saturation. The AW controller consists of a dynamic state feedback (DSF) controller and an AW compensator. A convex optimisation problem in terms of linear matrix inequalities is formulated to simultaneously design both the DSF controller and the AW compensator. The resulting AW controller depends on the singular perturbation parameter $\varepsilon$ and is shown to be well-conditioned for any $\varepsilon$ of interest. Furthermore, a two-stage design method is proposed to handle the case that $\varepsilon$ is unknown. An $\varepsilon$-independent DSF controller is designed at the first stage, and then an $\varepsilon$-independent AW compensator is constructed by solving a convex optimisation problem. Both of the methods can achieve a desired stability bound and enlarge the basin of attraction at the same time. Finally, examples are given to show the advantages and effectiveness of the obtained results.
\end{abstract}

\section{Introduction}

Actuator saturation is a common phenomenon in practical control systems and may lead to system performance's degradation or even instability if not properly accounted for in control design [1-4]. Thus intensive research efforts have been devoted to control systems subject to actuator saturation [5-9]. There are mainly two design strategies: direct design and indirect design. The former considers the actuation saturation at the outset of controller design [10-12], while the latter consists of an anti-windup (AW) compensator and a pre-designed controller achieving desired system performance when saturation does not occur. The indirect design approach attracts more attention of engineers because of its intuitive approach and abroad applicability. Since global stability of control systems with actuator saturation is difficult to achieve when the system is open-loop unstable [6], expanding the basin of attraction is still an open problem. Many of the indirect methods reported in the literature follow the two-step approach $[5,13]$ : first design a nominal controller without considering actuator saturation, then design the add-on AW compensator. This approach, however, often leads to conservatism in terms of performance and basin of attraction. Recently, the simultaneous design of the nominal controller and the AW compensator has been explored by several research groups, which brought a large basin of attraction and/or better performance $[14,15]$.

Singularly perturbed systems (SPSs), with a singular perturbation" parameter $\varepsilon$ determining the degree of separation between the slow and fast modes of the systems, often arise in engineered systems, such as aerospace systems, chemical processes, power systems and so on $[16,17]$. Stability problem of SPSs, which is different from that for normal systems, is known as the problem of determining a bound $\varepsilon_{0}$, such that stability of a given SPS is guaranteed for all $\varepsilon \in\left(0, \varepsilon_{0}\right]$ [18-21]. A key issue for stability analysis and control design of SPSs is to address the possible ill-conditioned numerical challenges resulted from the existence of singular perturbation parameter $\varepsilon$. In standard singular perturbation theory, the SPSs are decomposed into fast and slow reduced-order subsystems and then the analysis and design of the original system are reduced to the corresponding problems for the reduced-order systems. As a result, the ill-conditioned numerical issues are avoided and the computational burden is reduced $[16,17]$.
SPSs subject to actuator saturation are non-smooth systems, which violates one of the essential assumptions of the standard singular perturbation theory. Thus most available analysis and design methods based on reduced-order subsystems require additional conditions when actuator saturation is involved [22-25]. In [22], the saturation non-linearity is assumed to only rely on the slow state variables, while in $[23,24]$, the saturation non-linearity is required to only depend on the fast state variables. In [25], the reduced-order systems are constructed without taking saturation into account and a composite controller which can force the closed-loop system to have a linear behaviour in a region is designed. To alleviate these limits, alternative approaches not relying on system decomposition were proposed in $[26,27]$. These methods successfully avoid the possible ill-conditioned numerical issues by choosing appropriate Lyapunov functions at the cost of added computational burden. However, all of the preceding results are under the framework of the direct design. To the best knowledge of the authors, AW design (indirect design approach) is still an open problem for SPSs subject to actuator saturation.

In this paper, we will focus on the problem of AW controller design for SPSs subject to actuator saturation. An AW controller is constructed by combining a DSF controller and an AW compensator. We first consider the situation that the singular perturbation parameter $\varepsilon$ is known. By an $\varepsilon$-dependent Lyapunov function, simultaneous design of both parts of the AW controller is reduced to solving a convex optimisation problem in the form of LMIs. For the resulting AW controller, the DSF controller gains depend on $\varepsilon$, while the AW compensator gain does not. Since the controller takes advantage of the knowledge of the singular perturbation parameter $\varepsilon$, it can result in a desired stability bound and enlarge the basin of attraction at the same time. Then we pay attention to the case that the singular perturbation parameter $\varepsilon$ is unknown. A set of linear matrix inequalities (LMIs) are proposed for designing an $\varepsilon$-independent DSF controller. Based on the resulting DSF controller, an optimisation problem is formulated to design the AW compensator to achieve a desired stability bound while enlarging the basin of attraction. Finally, examples are presented to illustrate the proposed methods.

The rest of this paper is organised as follows: in Section 2, the problem under consideration is formulated and preliminaries are presented. In Sections 3 and 4, approaches to designing 
$\varepsilon$-dependent and $\varepsilon$-independent AW controllers are proposed, respectively. The designs are transformed into solving convex optimisation problems. Examples are given in Section 4 to illustrate various features of the proposed methods. Section 5 concludes the paper.

Notation: The superscript $\mathrm{T}$ stands for matrix transposition and the notation $M^{-\mathrm{T}}$ denotes the transpose of the inverse matrix of $M$. For vectors $v, w \in R^{p}, v \preceq w$ means that the inequalities between the vectors are componentwise. $\star$ denotes the block induced by symmetry. For a matrix $M, M_{(i)}$ denotes the $i$ th row of $M$.

\section{Problem formulation and preliminaries}

Consider the control system depicted in Fig. 1. The plant is a linear SPS represented by

$$
E(\varepsilon) \dot{x}(t)=A x(t)+B u_{m}(t)
$$

where $x \in R^{n}$ is the state, $u_{m} \in R^{q}$ is the control input,

$$
E(\varepsilon)=\left[\begin{array}{cc}
I_{n_{1}} & 0 \\
0 & \varepsilon I_{n_{2}}
\end{array}\right] \in R^{n \times n},
$$

$A \in R^{n \times n}$, and $B \in R^{n \times q}$ are constant matrices. The actuator is subject to saturation and thus the control input is generated by $u_{m}=\operatorname{sat}(u)$ with $u$ being the desired control input and sat( $(\cdot)$ being a componentwise saturation map $R^{q} \mapsto R^{q}$ defined as

$$
\operatorname{sat}\left(u_{i}(t)\right)=\operatorname{sign}\left(u_{i}(t)\right) \min \left\{1,\left|u_{i}(t)\right|\right\}, \quad i=1,2, \ldots, q .
$$

The goal of this paper is the design of an AW controller consisting of a DSF controller

$$
\dot{u}(t)=F x(t)+G u(t)+\zeta,
$$

and an AW compensator

$$
\zeta=E_{\mathrm{c}}(\operatorname{sat}(u(t))-u(t))
$$

where $u(t)$ is the controller state. Matrices $E_{\mathrm{c}} \in R^{q \times q}, F \in R^{q \times n}$ and $G \in R^{q \times q}$ are controller gains to be determined.

When the control input is not saturated, i.e. $\operatorname{sat}(u(t))=u(t)$, the AW controller will serve as a normal controller. The matrix $E_{\mathrm{c}}$ is AW compensator gain, and the matrices $F$ and $G$ are DSF controller gains.

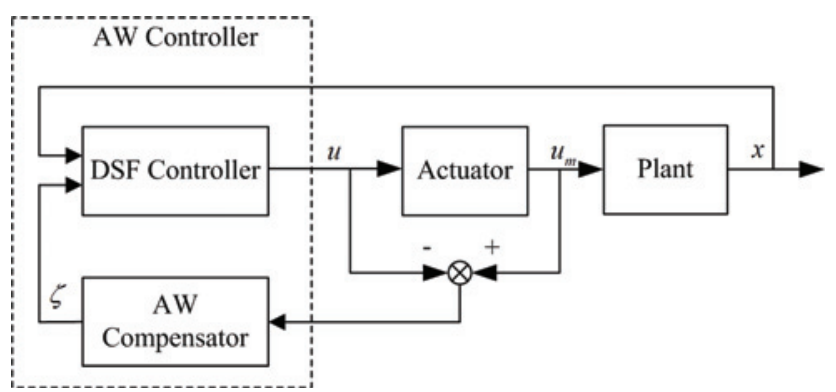

Fig. 1 AW close-loop system
The resulting closed-loop (1)-(4) is described as

$$
\tilde{E}(\varepsilon) \dot{\eta}(t)=\left(\hat{A}+I_{R} K\right) \eta+\left(\hat{B}+I_{R} E_{\mathrm{c}}\right) \psi(\hat{C} \eta),
$$

where $\psi(u(t))=\operatorname{sat}(u(t))-u(t)$ is a decentralised dead-zone nonlinearity, and

$$
\begin{aligned}
\eta & =\left[\begin{array}{l}
u \\
x
\end{array}\right], \quad \tilde{E}(\varepsilon)=\left[\begin{array}{cc}
I & 0 \\
0 & E(\varepsilon)
\end{array}\right], \quad \hat{A}=\left[\begin{array}{ll}
0 & 0 \\
B & A
\end{array}\right], \quad \hat{B}=\left[\begin{array}{l}
0 \\
B
\end{array}\right], \\
I_{R} & =\left[\begin{array}{l}
I \\
0
\end{array}\right], \quad \hat{C}=\left[\begin{array}{ll}
I & 0
\end{array}\right], \quad K=\left[\begin{array}{ll}
F & G
\end{array}\right] .
\end{aligned}
$$

The problem under consideration is expressed as:

Problem 1: Given a desired stability bound $\varepsilon_{0}>0$, determine an AW controller (3) and (4) and an ellipsoid $\Omega \subseteq R^{n}$, as large as possible, such that for any $\varepsilon \in\left(0, \varepsilon_{0}\right]$ the closed-loop system (5) is locally asymptotically stable with $\Omega$ contained in the basin of attraction.

The following lemmas will be used in the sequel:

Lemma 1 [28]: For any diagonal positive definite matrix $\Gamma \in R^{q \times q}$, the non-linearity $\psi(v)=\operatorname{sat}(v)-v$ satisfies the following inequality

$$
\psi^{\mathrm{T}}(v) \Gamma(\psi(v)+w) \leq 0, \quad \forall v, w \in S\left(v_{0}\right),
$$

where $S\left(v_{0}\right)=\left\{v, w \in R^{q} \mid-v_{0} \preceq v-w \preceq v_{0}\right\}$ and $v_{0} \in R^{q}$ is given.

Lemma 2 [29]: For a positive scalar $\varepsilon_{0}$ and symmetric matrices $S_{1}, S_{2}$ and $S_{3}$ with appropriate dimensions, if

$$
\begin{gathered}
S_{1} \geq 0, \\
S_{1}+\varepsilon_{0} S_{2}>0, \\
S_{1}+\varepsilon_{0} S_{2}+\varepsilon_{0}^{2} S_{3}>0,
\end{gathered}
$$

hold, then

$$
S_{1}+\varepsilon S_{2}+\varepsilon^{2} S_{3}>0, \quad \forall \varepsilon \in\left(0, \varepsilon_{0}\right]
$$

Lemma 3 [29]: If there exist matrices $Z_{i}(i=1,2, \ldots, 5)$ with $Z_{i}=$ $Z_{i}^{\mathrm{T}}(i=1,2,3,4)$ satisfying

$$
\begin{gathered}
Z_{1}>0 \\
{\left[\begin{array}{cc}
Z_{1}+\varepsilon_{0} Z_{3} & \varepsilon_{0} Z_{5}^{\mathrm{T}} \\
\varepsilon_{0} Z_{5} & \varepsilon_{0} Z_{2}
\end{array}\right]>0,} \\
{\left[\begin{array}{cc}
Z_{1}+\varepsilon_{0} Z_{3} & \varepsilon_{0} Z_{5}^{\mathrm{T}} \\
\varepsilon_{0} Z_{5} & \varepsilon_{0} Z_{2}+\varepsilon_{0}^{2} Z_{4}
\end{array}\right]>0,}
\end{gathered}
$$

then

$$
\tilde{E}(\varepsilon) Z(\varepsilon)=Z^{\mathrm{T}}(\varepsilon) \tilde{E}(\varepsilon)>0, \quad \forall \varepsilon \in\left(0, \varepsilon_{0}\right]
$$

where

$$
Z(\varepsilon)=\left[\begin{array}{cc}
Z_{1}+\varepsilon Z_{3} & \varepsilon Z_{5}^{\mathrm{T}} \\
Z_{5} & Z_{2}+\varepsilon Z_{4}
\end{array}\right]
$$

\section{$3 \quad$ Main results}

In this section, we will present two methods to solve Problem 1. First, we consider the case that the singular perturbation parameter $\varepsilon$ is known and can be used for controller design. An $\varepsilon$-dependent AW controller is designed. Then we come to the situation that $\varepsilon$ is unknown, and propose an $\varepsilon$-independent AW controller design. 


\subsection{Design of $\varepsilon$-dependent AW controller}

In this subsection, we present a convex optimisation problem to simultaneously design the DSF controller gains and the AW compensator gain. The following theorem establishes sufficient conditions for the existence of AW controller (3) and (4), under which the closed-loop system (5) is locally asymptotically stable for any singular perturbation parameter $\varepsilon$ of interest with a specified ellipsoid contained in the basin of attraction.

Theorem 1: Given a scalar $\varepsilon_{0}>0$, if there exist a diagonal positive definite matrix $S \in R^{q \times q}$, matrices $Q \in R^{q \times q}$, $M_{1} \in R^{q \times\left(n_{1}+q\right)}, \quad M_{2} \in R^{q \times n_{2}}, \quad Y \in R^{q \times n}, \quad Z_{1} \in R^{\left(n_{1}+q\right) \times\left(n_{1}+q\right)}$, $Z_{2} \in R^{n_{2} \times n_{2}}, Z_{3} \in R^{\left(n_{1}+q\right) \times\left(n_{1}+q\right)}, Z_{4} \in R^{n_{2} \times n_{2}}, Z_{5} \in R^{n_{2} \times\left(n_{1}+q\right)}$ with $Z_{i}=Z_{i}^{\mathrm{T}}(i=1,2,3,4)$, such that LMIs

$$
\begin{aligned}
& {\left[\begin{array}{cc}
\Phi & \star \\
S \hat{B}^{\mathrm{T}}+Q I_{R}^{\mathrm{T}}-M \tilde{E}(0)-\hat{C} U_{1} & -2 S
\end{array}\right]<0,} \\
& {\left[S \hat{B}^{\mathrm{T}}+Q I_{R}^{\mathrm{T}}-M \tilde{E}\left(\varepsilon_{0}\right)-\hat{C}\left(U_{1}+\varepsilon_{0} U_{2}\right) \quad-2 S\right]<0,} \\
& {\left[\begin{array}{cc}
Z_{1} & \star \\
M_{1(i)} & 1
\end{array}\right]>0, \quad i=1,2, \ldots, q,} \\
& {\left[\begin{array}{ccc}
Z_{1}+\varepsilon_{0} Z_{3} & \star & \star \\
\varepsilon_{0} Z_{5} & \varepsilon_{0} Z_{2} & \star \\
M_{1(i)} & \varepsilon_{0} M_{2(i)} & 1
\end{array}\right]>0, \quad i=1,2, \ldots, q,} \\
& {\left[\begin{array}{ccc}
Z_{1}+\varepsilon_{0} Z_{3} & \star & \star \\
\varepsilon_{0} Z_{5} & \varepsilon_{0} Z_{2}+\varepsilon_{0}^{2} Z_{4} & \star \\
M_{1(i)} & \varepsilon_{0} M_{2(i)} & 1
\end{array}\right]>0, \quad i=1,2, \ldots, q,}
\end{aligned}
$$

hold, where

$$
U_{1}=\left[\begin{array}{cc}
Z_{1} & 0 \\
Z_{5} & Z_{2}
\end{array}\right], \quad U_{2}=\left[\begin{array}{cc}
Z_{3} & Z_{5}^{\mathrm{T}} \\
0 & Z_{4}
\end{array}\right]
$$

$M=\left[\begin{array}{ll}M_{1} & M_{2}\end{array}\right], \Phi=U_{1}^{\mathrm{T}} \hat{A}^{\mathrm{T}}+\hat{A} U_{1}+Y^{\mathrm{T}} I_{R}^{\mathrm{T}}+I_{R} Y$ and $\Theta=\left(U_{1}+\right.$ $\left.\varepsilon_{0} U_{2}\right)^{\mathrm{T}} \hat{A}^{\mathrm{T}}+\hat{A}\left(U_{1}+\varepsilon_{0} U_{2}\right)+Y^{\mathrm{T}} I_{R}^{\mathrm{T}}+I_{R} Y$.

Then the AW controller (3) and (4) with $E_{\mathrm{c}}=Q^{\mathrm{T}} S^{-1}, K(\varepsilon)=$ $Y Z^{-1}(\varepsilon), Z(\varepsilon)=U_{1}+\varepsilon U_{2}$ stabilises the system (5) for any $\varepsilon \in\left(0, \varepsilon_{0}\right]$. And, the ellipsoid $\Omega(\varepsilon)=\left\{\eta \mid \eta^{\mathrm{T}} Z^{-\mathrm{T}}(\varepsilon) \tilde{E}(\varepsilon) \eta \leq 1\right\}$ is within the basin of attraction of the closed-loop system.

Proof: Let $v=\hat{C} \eta$ and $w=M \tilde{E}(\varepsilon) Z^{-1}(\varepsilon) \eta+\hat{C} \eta=\left(M \tilde{E}(\varepsilon) Z^{-1}\right.$ $(\varepsilon)+\hat{C}) \eta$. Then from Lemma 1 , the non-linearity $\psi(\hat{C} \eta)$ satisfies

$$
\psi^{\mathrm{T}}(\hat{C} \eta) \Gamma\left(\psi(\hat{C} \eta)+\left(M \tilde{E}(\varepsilon) Z^{-1}(\varepsilon)+\hat{C}\right) \eta\right) \leq 0, \quad \forall \eta \in S(\rho),
$$

where $\Gamma$ is an arbitrary diagonal positive definite matrix, $S(\rho)=$ $\left\{\eta \mid-\rho \preceq M \tilde{E}(\varepsilon) Z^{-1}(\varepsilon) \eta \preceq \rho\right\}$, and $\rho=\left[\begin{array}{llll}1 & 1 & \cdots & 1\end{array}\right]^{\mathrm{T}}$.

From Lemma 2, LMIs (17)-(19) imply that

$$
\left[\begin{array}{cc}
Z^{\mathrm{T}}(\varepsilon) \tilde{E}(\varepsilon) & \star \\
M_{(i)} \tilde{E}(\varepsilon) & 1
\end{array}\right]>0, \quad i=1,2, \ldots, q, \quad \forall \varepsilon \in\left(0, \varepsilon_{0}\right],
$$

which is equivalent to

$$
\left[\begin{array}{cc}
\tilde{E}^{-1}(\varepsilon) Z^{\mathrm{T}}(\varepsilon) & \star \\
M_{(i)} & 1
\end{array}\right]>0, \quad i=1,2, \ldots, q, \quad \forall \varepsilon \in\left(0, \varepsilon_{0}\right] .
$$

Pre- and post-multiplying (22) by

$$
\operatorname{diag}\left(\left[\tilde{E}^{-1}(\varepsilon) Z^{\mathrm{T}}(\varepsilon)\right]^{-1}, I\right)
$$

and its transpose, respectively, we have

$$
\left[\begin{array}{cc}
\tilde{E}(\varepsilon) Z^{-1}(\varepsilon) & \star \\
M_{(i)} \tilde{E}(\varepsilon) Z^{-1}(\varepsilon) & 1
\end{array}\right]>0, \quad i=1,2, \ldots, q,
$$

which implies

$$
\tilde{E}(\varepsilon) Z^{-1}(\varepsilon)>Z^{-\mathrm{T}}(\varepsilon) \tilde{E}(\varepsilon) M_{(i)}^{\mathrm{T}} M_{(i)} \tilde{E}(\varepsilon) Z^{-1}(\varepsilon) .
$$

Then for any $\eta \in \Omega(\varepsilon)$, it holds that

$$
\eta^{\mathrm{T}} Z^{-\mathrm{T}}(\varepsilon) \tilde{E}(\varepsilon) M_{(i)}^{\mathrm{T}} M_{(i)} \tilde{E}(\varepsilon) Z^{-1}(\varepsilon) \eta<1,
$$

which implies that $\Omega(\varepsilon) \subseteq S(\rho)$.

LMIs (15) and (16) imply

$\left[\begin{array}{cc}\Lambda & \star \\ S \hat{B}^{\mathrm{T}}+Q I_{R}^{\mathrm{T}}-M \tilde{E}(\varepsilon)-\hat{C}\left(U_{1}+\varepsilon U_{2}\right) & -2 S\end{array}\right]<0, \quad \forall \varepsilon \in\left(0, \varepsilon_{0}\right]$,

which can be rewritten as

$$
\left[\begin{array}{cc}
\Lambda & \star \\
S \hat{B}^{\mathrm{T}}+Q I_{R}^{\mathrm{T}}-M \tilde{E}(\varepsilon)-\hat{C} Z(\varepsilon) & -2 S
\end{array}\right]<0, \quad \forall \varepsilon \in\left(0, \varepsilon_{0}\right],
$$

where $\quad \Lambda=\left(U_{1}+\varepsilon U_{2}\right)^{\mathrm{T}} \hat{A}^{\mathrm{T}}+\hat{A}\left(U_{1}+\varepsilon U_{2}\right)+Y^{\mathrm{T}} I_{R}^{\mathrm{T}}+I_{R} Y=$ $Z^{\mathrm{T}}(\varepsilon) \hat{A}^{\mathrm{T}}+\hat{A} Z(\varepsilon)+Y^{\mathrm{T}} I_{R}^{\mathrm{T}}+I_{R} Y$.

Pre- and post-multiplying (24) by

$$
\operatorname{diag}\left(Z^{-\mathrm{T}}(\varepsilon), S^{-1}\right)
$$

and its transpose, respectively, we have

$$
\left[\begin{array}{cc}
\Phi_{1} & \star \\
\Phi_{2} & -2 S^{-1}
\end{array}\right]<0, \quad \forall \varepsilon \in\left(0, \varepsilon_{0}\right]
$$

where $\Phi_{1}=\hat{A}^{\mathrm{T}} Z^{-1}(\varepsilon)+Z^{-\mathrm{T}}(\varepsilon) Y^{\mathrm{T}} I_{R}^{\mathrm{T}} Z^{-1}(\varepsilon)+Z^{-\mathrm{T}}(\varepsilon) \hat{A}+Z^{-\mathrm{T}}$ $(\varepsilon) I_{R} Y Z^{-1}(\varepsilon)$ and $\Phi_{2}=\hat{B}^{\mathrm{T}} Z^{-1}(\varepsilon)+S^{-1} Q I_{R}^{\mathrm{T}} Z^{-1}(\varepsilon)-S^{-1} M \tilde{E}(\varepsilon)$ $Z^{-1}(\varepsilon)-S^{-1} \hat{C}$.

Letting $K(\varepsilon)=Y Z^{-1}(\varepsilon), \Gamma=S^{-1}, P(\varepsilon)=Z^{-1}(\varepsilon)$ and $E_{\mathrm{c}}=$ $Q^{\mathrm{T}} S^{-1}$ in (25), we have

$$
\begin{aligned}
\Pi \triangleq\left[\begin{array}{cc}
\Pi_{1} \\
\left(\hat{B}+I_{R} E_{\mathrm{c}}\right)^{\mathrm{T}} P(\varepsilon)-\Gamma M \tilde{E}(\varepsilon) P(\varepsilon)-\Gamma \hat{C} & -2 \Gamma
\end{array}\right]<0, \\
\quad \forall \varepsilon \in\left(0, \varepsilon_{0}\right],
\end{aligned}
$$

where $\Pi_{1}=\left(\hat{A}+I_{R} K(\varepsilon)\right)^{\mathrm{T}} P(\varepsilon)+P^{\mathrm{T}}(\varepsilon)\left(\hat{A}+I_{R} K(\varepsilon)\right)$.

By Lemma 3, LMIs (17)-(19) guarantee that

$$
\tilde{E}(\varepsilon) Z(\varepsilon)=Z^{\mathrm{T}}(\varepsilon) \tilde{E}(\varepsilon)>0, \quad \forall \varepsilon \in\left(0, \varepsilon_{0}\right],
$$

which implies

$$
\tilde{E}(\varepsilon) P(\varepsilon)=P^{\mathrm{T}}(\varepsilon) \tilde{E}(\varepsilon)>0, \quad \forall \varepsilon \in\left(0, \varepsilon_{0}\right] .
$$

Define an $\varepsilon$-dependent Lyapunov function

$$
V(\eta)=\eta^{\mathrm{T}} \tilde{E}(\varepsilon) P(\varepsilon) \eta .
$$

Computing the derivative of $V(\eta)$ along the trajectories of system (5) and taking into account (20) and (26), we have

$$
\begin{aligned}
\left.\dot{V}\right|_{(5)}= & (\tilde{E}(\varepsilon) \dot{\eta})^{\mathrm{T}} P(\varepsilon) \eta+\eta^{\mathrm{T}} P^{\mathrm{T}}(\varepsilon) \tilde{E}(\varepsilon) \dot{\eta} \\
= & \eta^{\mathrm{T}}\left(\left(\hat{A}+I_{R} K\right)^{\mathrm{T}} P(\varepsilon)+P^{\mathrm{T}}(\varepsilon)\left(\hat{A}+I_{R} K\right)\right) \eta \\
& +2 \psi^{\mathrm{T}}(\hat{C} \eta)\left(\hat{B}+I_{R} E_{\mathrm{c}}\right)^{\mathrm{T}} P(\varepsilon) \eta \\
\leq & \eta^{\mathrm{T}}\left(\left(\hat{A}+I_{R} K\right)^{\mathrm{T}} P(\varepsilon)+P^{\mathrm{T}}(\varepsilon)\left(\hat{A}+I_{R} K\right)\right) \eta \\
& +2 \psi^{\mathrm{T}}(\hat{C} \eta)\left(\hat{B}+I_{R} E_{\mathrm{c}}\right)^{\mathrm{T}} P(\varepsilon) \eta \\
& -2 \psi^{\mathrm{T}}(\hat{C} \eta) \Gamma(\psi(\hat{C} \eta)+(M \tilde{E}(\varepsilon) P(\varepsilon)+\hat{C}) \eta) \\
= & {\left[\begin{array}{c}
\eta \\
\psi
\end{array}\right]^{\mathrm{T}} \Pi\left[\begin{array}{l}
\eta \\
\psi
\end{array}\right]<0, \quad \forall \varepsilon \in\left(0, \varepsilon_{0}\right], \eta \in \Omega(\varepsilon), \eta \neq 0 . }
\end{aligned}
$$

Therefore, the closed-loop system is locally asymptotically stable for any $\varepsilon \in\left(0, \varepsilon_{0}\right]$. Moreover, the ellipsoid $\Omega(\varepsilon)$ is within the basin of attraction of the closed-loop system. 
Remark 1: For many SPSs, the singular perturbation parameter $\varepsilon$ has a physical meaning and its value is available for controller design. Thus various $\varepsilon$-dependent controllers have been designed for different kinds of SPSs [27, 29-31]. For such design problems, a key task is to ensure the design procedure and the obtained controller to be well-defined for any allowable singular perturbation parameter. Theorem 1 proposes sufficient conditions for the existence of $\varepsilon$-dependent AW controller (3) and (4). LMIs (15)-(19) depends on the stability bound $\varepsilon_{0}$, but not the singular perturbation parameter $\varepsilon$. It can be seen that LMI (16) becomes (15), while (18) and (19) are reduced to (17) when $\varepsilon_{0}$ is small enough. Thus LMIs (15)-(19) are well-conditioned.

Remark 2: From LMIs (17) and (18), it follows that $Z_{1}>0$ and $Z_{2}>0$, which implies that matrix

$$
U_{1}=\left[\begin{array}{cc}
Z_{1} & 0 \\
Z_{5} & Z_{2}
\end{array}\right]
$$

is non-singular. In addition, from the proof for Theorem 1 , it can be seen that $Z(\varepsilon)=U_{1}+\varepsilon U_{2}$ is non-singular for all $\varepsilon \in\left(0, \varepsilon_{0}\right]$. Therefore, $K(\varepsilon)=Y\left(U_{1}+\varepsilon U_{2}\right)^{-1}$ is well-defined for all $\varepsilon \in$ $\left(0, \varepsilon_{0}\right]$ and robust with respect to $\varepsilon$.

Remark 3: On analysis and design of control system with actuator saturation, there are two main approaches for dealing with saturation non-linearity, namely, the sector bound approach and the convex hull approach [6]. The convex hull approach is less conservative than the sector bound approach at the cost of computational complexity. When it comes to anti-windup design, the convex hull approach usually leads to bilinear matrix inequality problem, while the sector bound approach can be reduced to LMI problem which is much easier to solve [6,32]. Theorem 1 is derived by Lemma 1 which represents a modified sector bound approach. The convex hull approach can be applied to design the AW controller (3) and (4) by a similar way in $[32,33]$.

With the LMI conditions of Theorem 1, we are interested in obtaining the best estimate of the basin of attraction of the closedloop system. Following the line of [6], we try to maximise the volume of ellipsoid $\Omega(\varepsilon)$ by solving the following optimisation problem

$$
\begin{aligned}
& \min _{S, M, Y, U_{1}, U_{2}} \lambda \\
& \text { s.t. }(15)-(19), \\
& \qquad \lambda>0 \text { and } Z^{-\mathrm{T}}(\varepsilon) \tilde{E}(\varepsilon)<\lambda I .
\end{aligned}
$$

It can be seen that $Z^{-\mathrm{T}}(\varepsilon) \tilde{E}(\varepsilon)<\lambda I$ with $\lambda>0$ is equivalent to

$$
\left[\begin{array}{cc}
Z^{\mathrm{T}}(\varepsilon) \tilde{E}(\varepsilon) & \tilde{E}(\varepsilon) \\
\tilde{E}(\varepsilon) & \lambda I
\end{array}\right]<0
$$

By Lemma 2, inequality (31) is guaranteed by

$$
\begin{gathered}
{\left[\begin{array}{cccc}
Z_{1} & \star & \star & \star \\
0 & 0 & \star & \star \\
I & 0 & \lambda I & \star \\
0 & 0 & 0 & \lambda I
\end{array}\right] \geq 0,} \\
{\left[\begin{array}{cccc}
Z_{1}+\varepsilon_{0} Z_{3} & \star & \star & \star \\
\varepsilon_{0} Z_{5} & \varepsilon_{0} Z_{2} & \star & \star \\
I & 0 & \lambda I & \star \\
0 & \varepsilon_{0} I & 0 & \lambda I
\end{array}\right]>0,}
\end{gathered}
$$

and

$$
\left[\begin{array}{cccc}
Z_{1}+\varepsilon_{0} Z_{3} & \star & \star & \star \\
\varepsilon_{0} Z_{5} & \varepsilon_{0} Z_{2}+\varepsilon_{0}^{2} Z_{4} & \star & \star \\
I & 0 & \lambda I & \star \\
0 & \varepsilon_{0} I & 0 & \lambda I
\end{array}\right]>0 .
$$

It is easy to see that inequality (32) is equivalent to

$$
\left[\begin{array}{cc}
Z_{1} & \star \\
I & \lambda I
\end{array}\right]>0
$$

Then the optimisation problem (30) can be reformulated to the following convex optimisation problem

$$
\min _{S, M, Y, U_{1}, U_{2}} \lambda
$$

Remark 4: The convex optimisation problem (36) can be solved by numerical algorithms in polynomial time [34]. With the aid of LMI Toolbox in Matlab, we can solve the convex optimisation problem (36) efficiently.

\subsection{Design of $\varepsilon$-independent AW controller}

In this subsection, we first present a method for designing $\varepsilon$ independent DSF controller gains, and then formulate a convex optimisation problem to maximise the estimate of the basin of attraction by constructing an appropriate $\varepsilon$-independent antiwindup gain.

Theorem 2: If there exist a diagonal positive definite matrix $S \in R^{q \times q}$, matrices $Q \in R^{q \times q}, M_{1} \in R^{q \times\left(n_{1}+q\right)}, M_{2} \in R^{q \times n_{2}}, Y \in$ $R^{q \times n}, Z_{1} \in R^{\left(n_{1}+q\right) \times\left(n_{1}+q\right)}, Z_{2} \in R^{n_{2} \times n_{2}}, Z_{5} \in R^{n_{2} \times\left(n_{1}+q\right)}$ with $Z_{i}=$ $Z_{i}^{\mathrm{T}}(i=1,2)$, such that LMIs

$$
\begin{aligned}
& {\left[\begin{array}{cc}
\Phi & \star \\
S \hat{B}^{\mathrm{T}}+Q I_{R}^{\mathrm{T}}-M \tilde{E}(0)-\hat{C} U_{1} & -2 S
\end{array}\right]<0,} \\
& {\left[\begin{array}{cc}
Z_{1} & \star \\
M_{1(i)} & 1
\end{array}\right]>0, \quad i=1,2, \ldots, q,} \\
& {\left[\begin{array}{ccc}
Z_{1} & \star & \star \\
\alpha Z_{5} & \alpha Z_{2} & \star \\
M_{1(i)} & \alpha M_{2(i)} & 1
\end{array}\right]>0, \quad i=1,2, \ldots, q,}
\end{aligned}
$$

hold, where $\alpha$ is a pre-defined positive scalar,

$$
U_{1}=\left[\begin{array}{cc}
Z_{1} & 0 \\
Z_{5} & Z_{2}
\end{array}\right]
$$

$M=\left[\begin{array}{ll}M_{1} & M_{2}\end{array}\right]$ and $\Phi=U_{1}^{\mathrm{T}} \hat{A}^{\mathrm{T}}+\hat{A} U_{1}+Y^{\mathrm{T}} I_{R}^{\mathrm{T}}+I_{R} Y$.

Then there exists a positive scalar $\varepsilon_{0} \leq \alpha$, such that the closedloop system (5) with $K=\left[\begin{array}{ll}F & G\end{array}\right]=Y U_{1}^{-1}, E_{\mathrm{c}}=Q^{\mathrm{T}} S^{-1}$ is locally asymptotically stable for any $\varepsilon \in\left(0, \varepsilon_{0}\right]$. Moreover, the ellipsoid $\Omega\left(\varepsilon_{0}\right)=\left\{\eta \mid \eta^{\mathrm{T}} P\left(\varepsilon_{0}\right) \eta \leq 1\right\}$ with

$$
P\left(\varepsilon_{0}\right)=\left[\begin{array}{cc}
Z_{1} & Z_{5}^{\mathrm{T}} \\
Z_{5} & \frac{1}{\varepsilon_{0}} Z_{2}
\end{array}\right]^{-1}
$$

is within the basin of attraction of the closed-loop system.

Proof: LMI (39) implies that $Z_{2}>0$. Then by LMIs (37)-(39), there exist a positive scalar $\varepsilon_{0} \leq \alpha$, such that

$$
\begin{aligned}
& {\left[\begin{array}{cc}
\Theta & \star \\
S \hat{B}^{\mathrm{T}}+Q I_{R}^{\mathrm{T}}-M \tilde{E}\left(\varepsilon_{0}\right)-\hat{C}\left(U_{1}+\varepsilon_{0} U_{2}\right) & -2 S
\end{array}\right]<0,} \\
& {\left[\begin{array}{ccc}
Z_{1} & \star & \star \\
\varepsilon_{0} Z_{5} & \varepsilon_{0} Z_{2} & \star \\
M_{1(i)} & \varepsilon_{0} M_{2(i)} & 1
\end{array}\right]>0, \quad i=1,2, \ldots, q,}
\end{aligned}
$$

where

$$
\begin{gathered}
U_{2}=\left[\begin{array}{cc}
0 & Z_{5}^{\mathrm{T}} \\
0 & 0
\end{array}\right], \\
\text { and } \Theta=\left(U_{1}+\varepsilon_{0} U_{2}\right)^{\mathrm{T}} \hat{A}^{\mathrm{T}}+\hat{A}\left(U_{1}+\varepsilon_{0} U_{2}\right)+Y^{\mathrm{T}} I_{R}^{\mathrm{T}}+I_{R} Y .
\end{gathered}
$$


Then using Theorem 1 by setting $Z_{4}=0$, the inequalities (37), (38), (40) and (41) yield that the closed-loop system (5) with $K=$ $\left[\begin{array}{ll}F & G\end{array}\right]=Y U_{1}^{-1}, E_{\mathrm{c}}=Q^{\mathrm{T}} S^{-1}$ is locally asymptotically stable for any $\varepsilon \in\left(0, \varepsilon_{0}\right]$. Moreover, the ellipsoid $\Omega(\varepsilon)=\left\{\eta \mid \eta^{\mathrm{T}} P(\varepsilon) \eta \leq 1\right\}$ with

$$
P(\varepsilon)=\left[\begin{array}{ll}
Z_{1} & Z_{5}^{\mathrm{T}} \\
Z_{5} & \frac{1}{\varepsilon} Z_{2}
\end{array}\right]^{-1}
$$

is an estimate of the basin of attraction of the closed-loop system. In addition, it follows from (41) that

$$
\left[\begin{array}{cc}
Z_{1} & Z_{5}^{\mathrm{T}} \\
Z_{5} & \frac{1}{\varepsilon_{0}} Z_{2}
\end{array}\right]>0
$$

Inequality (42) implies that

$$
\left[\begin{array}{cc}
Z_{1} & Z_{5}^{\mathrm{T}} \\
Z_{5} & \frac{1}{\varepsilon} Z_{2}
\end{array}\right] \geq\left[\begin{array}{cc}
Z_{1} & Z_{5}^{\mathrm{T}} \\
Z_{5} & \frac{1}{\varepsilon_{0}} Z_{2}
\end{array}\right], \quad \forall \varepsilon \in\left(0, \varepsilon_{0}\right],
$$

which is equivalent to

$$
\left[\begin{array}{cc}
Z_{1} & Z_{5}^{\mathrm{T}} \\
Z_{5} & \frac{1}{\varepsilon} Z_{2}
\end{array}\right]^{-1} \leq\left[\begin{array}{cc}
Z_{1} & Z_{5}^{\mathrm{T}} \\
Z_{5} & \frac{1}{\varepsilon_{0}} Z_{2}
\end{array}\right]^{-1}, \quad \forall \varepsilon \in\left(0, \varepsilon_{0}\right] .
$$

Then we have $\Omega\left(\varepsilon_{0}\right) \subseteq \Omega(\varepsilon), \forall \varepsilon \in\left(0, \varepsilon_{0}\right]$. That is, the ellipsoid $\Omega\left(\varepsilon_{0}\right)$ is within the basin of attraction of the closed-loop system for any $\varepsilon \in\left(0, \varepsilon_{0}\right]$. This completes the proof.

Remark 5: In the proof of Theorem 2, LMI (39) is not involved, which means that the conclusion of Theorem 2 still hold even if LMI (39) is omitted. We notice that LMIs (37) and (38) with $Z_{2}>$ 0 are sufficient conditions for the existence of AW controllers, but do not take into account the enlargement of the stability bound and basin of attraction. To get satisfactory stability bound and basin of attraction, we introduce LMI (39) which is closely related with the stability bound and the structure of basin of attraction.

By Theorem 2, we can get an $\varepsilon$-independent AW controller, under which the closed-loop system is locally asymptotically stable for small enough $\varepsilon$. With the obtained $\varepsilon$-independent gains $F$ and $G$, the following theorem presents a method for constructing a new AW compensator gain $E_{\mathrm{c}}$ to achieve a desired stability bound and enlarge the basin of attraction.

Theorem 3: Given a pre-defined stability bound $\varepsilon_{0}>0$, and DSF controller gains $F$ and $G$, if there exist a diagonal positive definite matrix $S \in R^{q \times q}$, matrices $Q \in R^{q \times q}, M_{1} \in R^{q \times\left(n_{1}+q\right)}$, $M_{2} \in R^{q \times n_{2}}, \quad Y \in R^{q \times n}, \quad Z_{1} \in R^{\left(n_{1}+q\right) \times\left(n_{1}+q\right)}, Z_{2} \in R^{n_{2} \times n_{2}}, \quad Z_{5} \in$ $R^{n_{2} \times\left(n_{1}+q\right)}$ with $Z_{i}=Z_{i}^{\mathrm{T}}(i=1,2)$, such that LMIs

$$
\begin{aligned}
& {\left[\begin{array}{cc}
\Phi & \star \\
S \hat{B}^{\mathrm{T}}+Q I_{R}^{\mathrm{T}}-M \tilde{E}(0)-\hat{C} U_{1} & -2 S
\end{array}\right]<0,} \\
& {\left[\begin{array}{cc}
\Theta \hat{B}^{\mathrm{T}}+Q I_{R}^{\mathrm{T}}-M \tilde{E}\left(\varepsilon_{0}\right)-\hat{C}\left(U_{1}+\varepsilon_{0} U_{2}\right) & -2 S
\end{array}\right]<0,} \\
& {\left[\begin{array}{cc}
Z_{1} & \star \\
M_{1(i)} & 1
\end{array}\right]>0, \quad i=1,2, \ldots, q,} \\
& {\left[\begin{array}{ccc}
Z_{1} & \star & \star \\
\varepsilon_{0} Z_{5} & \varepsilon_{0} Z_{2} & \star \\
M_{1(i)} & \varepsilon_{0} M_{2(i)} & 1
\end{array}\right]>0, \quad i=1,2, \ldots, q,}
\end{aligned}
$$

hold, where

$$
U_{1}=\left[\begin{array}{cc}
Z_{1} & 0 \\
Z_{5} & Z_{2}
\end{array}\right], \quad U_{2}=\left[\begin{array}{cc}
0 & Z_{5}^{\mathrm{T}} \\
0 & 0
\end{array}\right],
$$

$M=\left[\begin{array}{ll}M_{1} & M_{2}\end{array}\right]$ and $\Phi=U_{1}^{\mathrm{T}}\left(\hat{A}+I_{R} K\right)^{\mathrm{T}}+\left(\hat{A}+I_{R} K\right) U_{1}$ and $\Theta=$ $\left(U_{1}+\varepsilon_{0} U_{2}\right)^{\mathrm{T}}\left(\hat{A}+I_{R} K\right)^{\mathrm{T}}+\left(\hat{A}+I_{R} K\right)\left(U_{1}+\varepsilon_{0} U_{2}\right)$.
Then the closed-loop system (5) with $K=\left[\begin{array}{ll}F & G\end{array}\right], E_{\mathrm{c}}=$ $Q^{\mathrm{T}} S^{-1}$ is locally asymptotically stable for any $\varepsilon \in\left(0, \varepsilon_{0}\right]$. Moreover, the ellipsoid $\Omega\left(\varepsilon_{0}\right)=\left\{\eta \mid \eta^{\mathrm{T}} P\left(\varepsilon_{0}\right) \eta \leq 1\right\}$ with

$$
P\left(\varepsilon_{0}\right)=\left[\begin{array}{cc}
Z_{1} & Z_{5}^{\mathrm{T}} \\
Z_{5} & \frac{1}{\varepsilon_{0}} Z_{2}
\end{array}\right]^{-1}
$$

is within the basin of attraction of the closed-loop system.

Using Theorem 3 and similar to the last subsection, the following convex optimisation problem can be used to maximise the estimate of the basin of attraction

$$
\begin{aligned}
& \min _{S, M, Y, U_{1}, U_{2}} \lambda \\
& \text { s.t. }(43)-(46) \text { and } \\
& {\left[\begin{array}{cc}
Z_{1} & \star \\
I & \lambda I
\end{array}\right]>0,} \\
& {\left[\begin{array}{cccc}
Z_{1} & \star & \star & \star \\
\varepsilon_{0} Z_{5} & \varepsilon_{0} Z_{2} & \star & \star \\
I & 0 & \lambda I & \star \\
0 & \varepsilon_{0} I & 0 & \lambda I
\end{array}\right]>0 .}
\end{aligned}
$$

Remark 6: The approach proposed in the previous subsection takes advantage of the knowledge of the singular perturbation parameter $\varepsilon$ and designs the DSF controller and AW compensator simultaneously. This subsection presents a two-stage method for designing $\varepsilon$-independent $\mathrm{AW}$ controller. The $\varepsilon$-dependent $\mathrm{AW}$ controller can result in larger stability bound and estimate of basin of attraction while $\varepsilon$-independent AW controller can be applied to the case that $\varepsilon$ is unknown.

\section{Examples}

In this section, two examples are given to illustrate various features of the proposed methods and show their advantages over the existing results.

Example 1: This example will demonstrate the advantage of the $\varepsilon$-dependent AW controller over the existing results. Consider an inverted pendulum system controlled by a DC motor via a gear train. The model, which was first established in [35], is described by

$$
\left\{\begin{array}{l}
\dot{x}_{1}(t)=x_{2}(t), \\
\dot{x}_{2}(t)=\frac{g}{l} \sin \left(x_{1}(t)\right)+\frac{N K_{\mathrm{m}}}{m l^{2}} x_{3}(t), \\
L_{a} \dot{x}_{3}(t)=-K_{\mathrm{b}} N x_{2}(t)-R_{a} x_{3}(t)+u(t),
\end{array}\right.
$$

where $x_{1}(t)=\theta_{\mathrm{p}}(t)$ denotes the angle $(\mathrm{rad})$ of the pendulum from the vertical upward, $x_{2}(t)=\dot{\theta}_{\mathrm{p}}(t), x_{3}(t)=I_{a}(t)$ denotes the current of the motor, $u(t)$ is the control input voltage, $K_{\mathrm{m}}$ is the motor torque constant, $K_{\mathrm{b}}$ is the back emf constant, $N$ is the gear ratio and $L_{a}$ is the inductance which is usually a small positive constant.

The parameters for the plant are as follows: $g=9.8 \mathrm{~m} / \mathrm{s}^{2}, N=$ $50, l=1 \mathrm{~m}, m=1 \mathrm{~kg}, K_{\mathrm{m}}=0.1 \mathrm{Nm} / \mathrm{A}, K_{\mathrm{b}}=0.1 \mathrm{Vs} / \mathrm{rad}, R_{a}=$ $1 \Omega$ and $L_{a}=0.05 \mathrm{H}$ and the input voltage is required to satisfy $|u| \leq 1$. Note that $L_{a}$ represents the singular perturbation parameter of the system. With these parameters, the linearised system of (48) is as follows

$$
\left\{\begin{array}{l}
\dot{x}_{1}(t)=x_{2}(t), \\
\dot{x}_{2}(t)=9.8 x_{1}(t)+x_{3}(t), \\
\varepsilon \dot{x}_{3}(t)=-x_{2}(t)-x_{3}(t)+u
\end{array}\right.
$$

where $\varepsilon=L_{a}$. 
The equilibrium point of system (49), that is, $x_{e}=\left[\begin{array}{lll}0 & 0 & 0\end{array}\right]^{\mathrm{T}}$ corresponds to the upright rest position of the inverted pendulum. We will design a controller to balance the pendulum around its upright rest position.

For system (49), the following static state feedback controller was proposed in [27]

$$
u=\left[\begin{array}{lll}
-2920.1 & -813.0 & -44.6
\end{array}\right] x
$$

and the obtained estimate of the basin of attraction is $\Omega_{1}=\{x \in$ $\left.R^{3} \mid x^{\mathrm{T}} P_{1} x \leq 1\right\}$, with

$$
P_{1}=\left[\begin{array}{ccc}
153.7649 & 42.9750 & 1.8181 \\
42.9750 & 12.0363 & 0.5066 \\
1.8181 & 0.5066 & 0.0291
\end{array}\right]
$$

We now use Theorem 1 to design an AW controller (3) and (4) for system (49). To do this, we first augment system (49) into the form of (5). The obtained system matrices are as follows

$$
\begin{gathered}
\tilde{E}(\varepsilon)=\left[\begin{array}{llll}
1 & 0 & 0 & 0 \\
0 & 1 & 0 & 0 \\
0 & 0 & 1 & 0 \\
0 & 0 & 0 & \varepsilon
\end{array}\right], \quad \hat{A}=\left[\begin{array}{cccc}
0 & 0 & 0 & 0 \\
0 & 0 & 1 & 0 \\
0 & 9.8 & 0 & 1 \\
1 & 0 & -1 & -1
\end{array}\right], \\
\hat{B}=\left[\begin{array}{l}
0 \\
0 \\
0 \\
1
\end{array}\right], \quad I_{R}=\left[\begin{array}{l}
1 \\
0 \\
0 \\
0
\end{array}\right], \quad \hat{C}=\left[\begin{array}{llll}
1 & 0 & 0 & 0
\end{array}\right] .
\end{gathered}
$$

Solving the optimisation problem (36) with $\varepsilon_{0}=0.1$, we have

$$
\begin{aligned}
& Z_{1}=\left[\begin{array}{ccc}
1079.1000 & -1.0000 & 2.5000 \\
-1.0000 & 4.2000 & -15.2000 \\
2.5000 & -15.2000 & 55.0000
\end{array}\right] \text {, } \\
& Z_{3}=\left[\begin{array}{ccc}
4.8711 & -2.7063 & 8.7456 \\
-2.7063 & 7.2440 & -19.5834 \\
8.7456 & -19.5834 & 52.6286
\end{array}\right] \text {, } \\
& Z_{2}=7.3379, Z_{4}=-0.6264, Z_{5}=\left[\begin{array}{lll}
1.6524 & -4.38449 .4953
\end{array}\right] \text {, } \\
& Y=\left[\begin{array}{llll}
-427.9000 & 0.8000 & -1.6000 & -1077.8000
\end{array}\right], \\
& M_{1}=\left[\begin{array}{lll}
-3.0688 & 0.0152 & 0.2274
\end{array}\right], \quad M_{2}=-0.0895 \text {, } \\
& S=0.7323, Q=1076.1000, \lambda=181.6076 .
\end{aligned}
$$

Taking into account $\varepsilon=0.05$, the $\mathrm{AW}$ controller gains are as follows

$$
\begin{aligned}
K & =\left[\begin{array}{ll}
F & G
\end{array}\right] \\
& =\left[\begin{array}{llll}
-9.0000 & -39419.0000 & -10988.0000 & -616.0000
\end{array}\right], \\
E_{\mathrm{c}} & =1469.4000 .
\end{aligned}
$$

An estimate of the basin of attraction of the augmented closed-loop system is $\Omega=\left\{\eta \in R^{4} \mid \eta^{\mathrm{T}} P \eta \leq 1\right\}$, where

$$
P=\left[\begin{array}{cccc}
0.0009 & 0.0358 & 0.0100 & 0.0004 \\
0.0358 & 154.5060 & 43.1649 & 1.8306 \\
0.0100 & 43.1649 & 12.0767 & 0.5103 \\
0.0004 & 1.8306 & 0.5103 & 0.0286
\end{array}\right]
$$

To compare the ellipsoids $\Omega_{1}$ and $\Omega$ intuitively, we make the projection of $\Omega$ on the coordinates $x$, which leads to an ellipsoid $\Omega_{2}=\left\{x \in R^{3} \mid x^{\mathrm{T}} P_{2} x \leq 1\right\}$. According to Lemma 5 of [24],

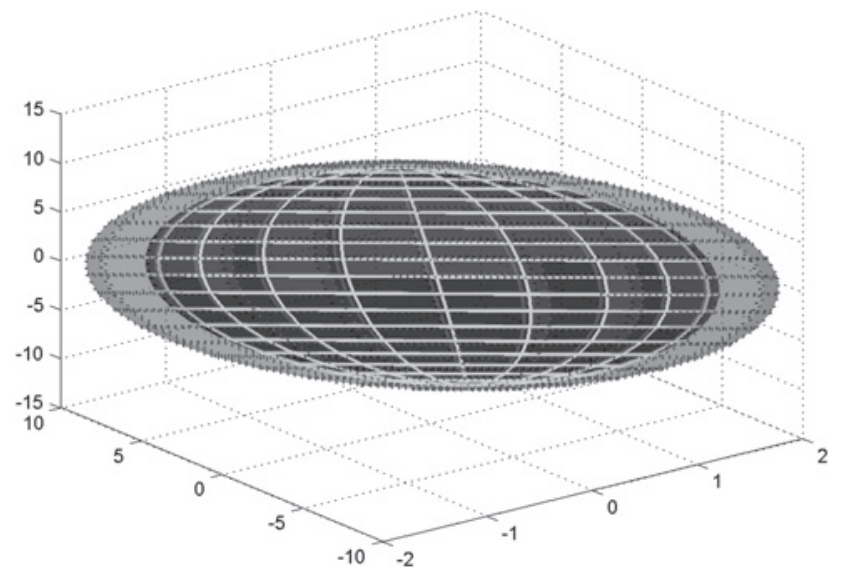

Fig. 2 Comparison of the ellipsoids $\Omega_{1}$ and $\Omega_{2}$

$$
\begin{aligned}
P_{2}=P_{22} & -P_{12}^{\mathrm{T}} P_{11}^{-1} P_{12} \text { with } \\
P_{11} & =0.0009, \quad P_{12}=\left[\begin{array}{lll}
0.0358 & 0.0100 & 0.0004
\end{array}\right], \\
P_{22} & =\left[\begin{array}{ccc}
154.5060 & 43.1649 & 1.8306 \\
43.1649 & 12.0767 & 0.5103 \\
1.8306 & 0.5103 & 0.0286
\end{array}\right] .
\end{aligned}
$$

By simple calculation, we have

$$
P_{2}=\left[\begin{array}{ccc}
153.0820 & 42.7671 & 1.8147 \\
42.7671 & 11.9656 & 0.5059 \\
1.8147 & 0.5059 & 0.0284
\end{array}\right]
$$

Fig. 2 depicts the ellipsoids $\Omega_{1}$ and $\Omega_{2}$, which shows that the estimate of the basin of attraction obtained by Theorem 1 is larger than that given by Yang et al. [27].

Example 2: This example will show how the two-stage method in Section 3.2 is applied to the inverted pendulum system (49). When the singular perturbation parameter $\varepsilon$ is not known, the AW controller designed by Theorem 1 cannot be used to stabilise the system. The two-stage method in Section 3.2 is useful in this case.

First, Theorem 2 is used to construct $\varepsilon$-independent DSF controller gains. Solving LMIs (37)-(39)with $\alpha=0.1$ leads to $\varepsilon$-independent DSF controller gains

$$
K=\left[\begin{array}{ll}
F & G
\end{array}\right]=\left[\begin{array}{llll}
-3.0067 & -1718.1357 & -489.5623 & -34.1096
\end{array}\right] .
$$

Then, with the obtained gain matrix $K$, the optimisation problem (47) is used to design $\varepsilon$-independent AW compensator gain to achieve a desired stability bound and enlarge the estimate of basin of attraction. Solving the optimisation problem (47) with the obtained matrix $K$ and $\varepsilon_{0}=0.05$ results in the AW compensator gain $E_{\mathrm{c}}=149.8265$ and an estimate of basin of attraction $\hat{\Omega}=\left\{\eta \in R^{4} \mid \eta^{\mathrm{T}} \hat{P} \eta \leq 1\right\}$, where

$$
\hat{P}=\left[\begin{array}{cccc}
0.0874 & 1.6460 & 0.4349 & -0.0431 \\
1.6460 & 419.1652 & 119.2192 & 3.3353 \\
0.4349 & 119.2192 & 34.3254 & 0.9759 \\
-0.0431 & 3.3353 & 0.9759 & 0.1750
\end{array}\right] \text {. }
$$

According to Theorem 3, the closed-loop system is locally asymptotically stable for any $\varepsilon \in(0,0.05]$ and the ellipsoid $\hat{\Omega}$ is within the basin of attraction. It is easy to show that $\eta_{0}=$ $\left[\begin{array}{llll}0 & -0.43 & 1.5 & -0.75\end{array}\right]^{\mathrm{T}} \in \hat{\Omega}$. Figs. 3 and 4 depict the state response and control signal starting from $\eta_{0}$ for $\varepsilon=0.03$ and $\varepsilon=0.05$, respectively. It can be seen that the trajectories converge to the origin in both cases. 


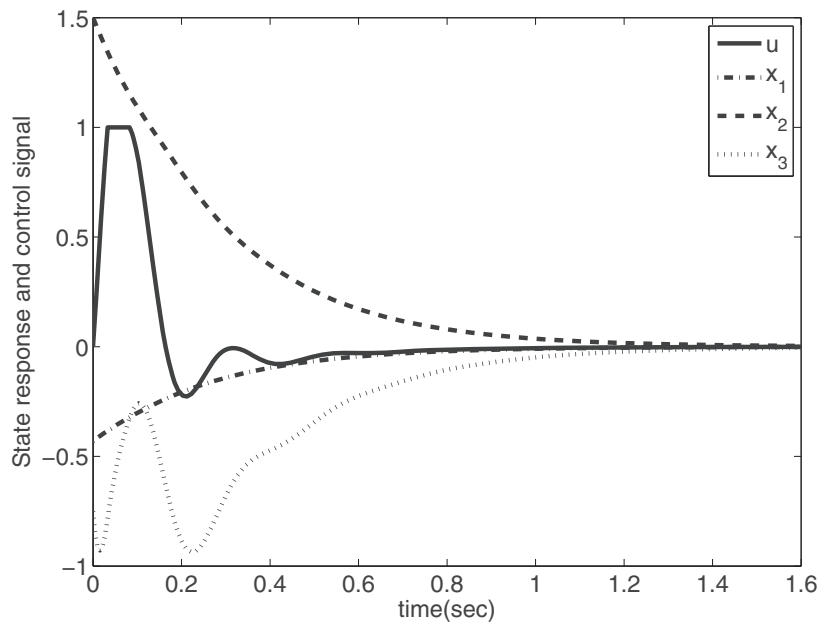

Fig.3 State response and control signal with $\varepsilon=0.03$

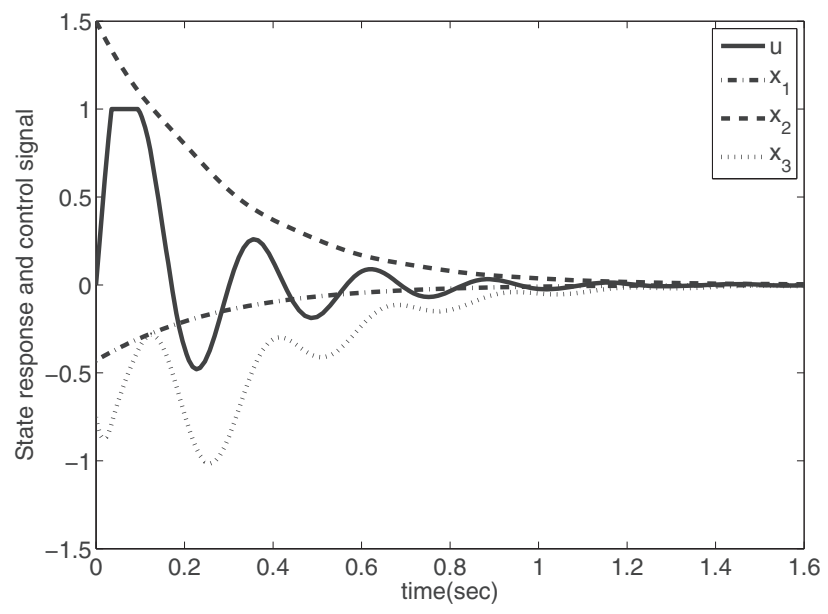

Fig. 4 State response and control signal with $\varepsilon=0.05$

When we disregard LMI condition (39) in the first stage, we get an estimate of the basin of attraction $\bar{\Omega}=\left\{\eta \in R^{4} \mid \eta^{\mathrm{T}} \bar{P} \eta \leq 1\right\}$ corresponding to stability bound $\varepsilon_{0}=0.05$, where

$$
\bar{P}=\left[\begin{array}{cccc}
0.2824 & 12.8211 & 3.6714 & -0.0682 \\
12.8211 & 2893.1759 & 793.6079 & 6.3982 \\
3.6714 & 793.6079 & 223.4684 & 1.7982 \\
-0.0682 & 6.3982 & 1.7982 & 0.3969
\end{array}\right]
$$

It is easy to see that the ellipsoid $\bar{\Omega}$ is much smaller than $\hat{\Omega}$. Thus LMI condition (39) plays a key role in the two-stage design.

\section{Conclusion}

This paper presents approaches to design AW controllers consisting of a dynamic state feedback controller and an AW compensator for SPSs subject to actuator saturation. When the singular perturbation parameter $\varepsilon$ is known, the two parts of the AW controller can be designed simultaneously by solving a convex optimisation problem. When $\varepsilon$ is unknown, the dynamic state feedback controller and AW compensator can be designed sequentially. Both designs can lead to a desired stability bound and enlarge the basin of attraction of the closed-loop systems. The presented examples have illustrated the proposed methods.

\section{Acknowledgments}

This work was supported by the National Natural Science Foundation of China (61374043), the Jiangsu Provincial Natural Science Foundation of China (BK20130205), the China Postdoctoral Science Foundation funded project (2013M530278, 2014T70558), the Fundamental Research Funds for the Central Universities (2013QNA50, 2013RC10, 2013RC12, 2013XK09) and the Natural Science Foundation of Liaoning Province (201202201).

\section{References}

1 Lin, Z., Saberi, A.: 'Semi-global exponential stabilization of linear systems subject to input saturation via linear feedbacks', Syst. Control Lett., 1993, 21, (1), pp. $225-239$

2 Wei, Y.L., Zheng, W.X., Li, Z., et al.: 'Anti-windup design of uncertain discretetime Markovian jump systems with partially known transition probabilities and saturating actuator', Int. J. Syst. Sci., 2015, 46, (7), pp. 1288-1298

3 Yang, H.J., Li, Z.W., Hua, C.C., et al.: 'Stability analysis of delta operator systems with actuator saturation by a saturation-dependent Lyapunov function', Circuits Syst. Signal Process., 2015, 34, (3), pp. 971-986

4 Yang, H.J., Li, H.B., Sun, F.C., et al.: 'Robust control for Markovian jump delta operator systems with actuator saturation', Eur. J. Control, 2014, 20, (4), pp. 207-215

5 Cao, Y.Y., Lin, Z., Ward, D.G., et al.: 'An antiwindup approach to enlarging domain of attraction for linear systems subject to actuator saturation', IEEE Trans. Autom. Control, 2002, 47, (1), pp. 140-145

$6 \mathrm{Hu}, \mathrm{T} ., \mathrm{Lin}, \mathrm{Z}$.: 'Control systems with actuator saturation: analysis and design' (Birkhäuser, Boston, MA, 2001)

7 Zhou, B., Duan, G.R., Lin, Z.L.: 'A parametric periodic Lyapunov equation with application in semi-global stabilization of discrete-time. Periodic systems subject to actuator saturation', Automatica, 2011, 47, (2), pp. 316-325

8 Yang, H.J., Li, X., Liu, Z.X., et al.: 'Robust fuzzy-scheduling control for nonlinear systems subject to actuator saturation via delta operator approach', Inf. Sci., 2014, 272, pp. 158-172

9 Yang, H.J., Shi, P., Li, Z.W., et al.: 'Analysis and design for delta operator systems with actuator saturation', Int. J. Control, 2014, 87, (5), pp. 987-999

10 Gutman, P.O., Hagander, P.: 'A new design of constrained controllers for linear systems', IEEE Trans. Autom. Control, 1985, 30, (1), pp. 22-33

11 Sussmann, H.J., Sontag, E.D., Yang, Y.: 'A general result on the stabilization of linear systems using bounded controls', IEEE Trans. Autom. Control, 1994, 39, (12), pp. 2411-2425

12 Hu, T., Teel, A.R., Zaccarian, L.: 'Stability and performance for saturated systems via quadratic and nonquadratic Lyapunov functions', IEEE Trans. Autom. Control, 2006, 51, (11), pp. 1770-1786

$13 \mathrm{Lu}, \mathrm{L}$. , Lin, Z.: 'Design of switched linear systems in the presence of actuator saturation', IEEE Trans. Autom. Control, 2008, 53, (6), pp. 1536-1542

14 Zhang, T., Feng, G., Liu, H., et al: 'Piecewise fuzzy anti-windup dynamic output feedback control of nonlinear processes with amplitude and rate actuator saturations', IEEE Trans. Fuzzy Syst., 2009, 17, (2), pp. 253-264

15 Sawada, K., Kiyama, T., Iwasaki, T.: 'Generalized sector synthesis of output feedback control with anti-windup structure', Syst. Control Lett., 2009, 58, (6), pp. $421-428$

16 Kokotovic, P.V., Khalil, H.K., O'Reilly, J.: 'Singular perturbation methods in control: analysis and design' (Philadelphia, PA: SIAM, 1999)

17 Khalil, H.: 'Stability analysis of nonlinear multiparameter singularly perturbed systems', IEEE Trans. Autom. Control, 1987, 32, (3), pp. 260-263

18 Cao, L., Schwartz, H.M. 'Complementary results on the stability bounds of singularly perturbed systems', IEEE Trans. Autom. Control, 2004, 49, (11), pp. 2017-2021

19 Saydy, L.: 'New stability/performance results for singularly perturbed systems', Automatica, 1996, 32, (6), pp. 807-818

20 Li, T.H.S., Li, J.H.: 'Stabilization bound of discrete two-time-scale systems', Syst. Control Lett., 1992, 18, (6), pp. 479-489

21 Chiou, J.S., Kung, F.C., Li, T.H.S.: 'An infinite $\varepsilon$-bound stabilization design for a class of singularly perturbed systems', IEEE Trans. Circuits Syst. I, Fundam. Theory Appl., 1999, 46, (12), pp. 1507-1510

22 Liu, P.L.: 'Stabilization of singularly perturbed multiple-time-delay systems with a saturating actuator', Int. J. Syst. Sci., 2001, 32, (8), pp. 1041-1045

23 Xin, H., Wu, D., Gan, D.Q., et al.: 'A method for estimating the stability region of singular perturbation systems with saturation nonlinearities', Aata Autam. Sin., 2008, 34, (12), pp. 1549-1555

24 Xin, H., Gan, D., Huang, M., et al.: 'Estimating the stability region of singular perturbation power systems with saturation nonlinearities: an linear matrix inequality based method', IET Control Theory Appl., 2010, 4, (3), pp. 351-361

25 Garcia, G., Tarbouriech, S. 'Control of singularly perturbed systems by bounded control', Proc. American Control Conf., Denver, Colorado, June 2003, pp. 4482 4487

26 Lizarraga, I., Tarbouriech, S., Garcia, G.: 'Control of singularly perturbed systems under actuator saturation', 16th IFAC World Congress, Prague, Czech Republic, July 2005, vol. 16, pp. 243-248

27 Yang, C.Y., Sun, J., Ma, X.P.: 'Stabilization bound of singularly perturbed systems subject to actuator saturation', Automatica, 2013, 49, (2), pp. 457-462

28 Tarbouriech, S., Prieur, C., Gomes da Silva, J.M. Jr.: 'Stability analysis and stabilization of systems presenting nested saturations', IEEE Trans. Autom. Control, 2006, 51, (8), pp. 1364-1371 
29 Yang, C., Zhang, Q.: 'Multi-objective control for T-S fuzzy singularly perturbed systems', IEEE Trans. Fuzzy Syst., 2009, 17, (1), pp. $104-115$

30 Datta, K.B., Raichaudhuri, A.: ' $H_{2} / H_{\infty}$ Control of discrete singularly perturbed systems: the state feedback case', Automatica, 2002, 38, (10), pp. 17911797

31 Assawinchaichote, W., Nguang, S.K., Shi, P.: ' $H_{\infty}$ output feedback control design for uncertain fuzzy singularly perturbed systems: an LMI approach', Automatica, 2004, 40, (12), pp. 2147-2152
32 Li, Y., Lin, Z.: 'Design of saturation-based switching anti-windup gains for the enlargement of the domain of attraction', IEEE Trans. Autom. Control, 2013 58, (7), pp. 1810-1816

33 Li, Y., Lin, Z.: 'Saturation-based switching anti-windup design for linear systems with nested input saturation', Automatica, 2014, 50, (11), pp. 2888-2896

34 Boyd, S., Ghaoui, L. E., Feron, E., et al.: 'Linear matrix inequalities in system and control theory' (Philadelphia, PA: SIAM, 1994)

35 Zak, S.H., Maccarley, C.A.: 'State-feedback control of non-linear systems', Int J. Control, 1986, 43, (5), pp. 1497-1514 